\title{
Animal Models of Tinnitus: A Review
}

\author{
Alexander Galazyuk ${ }^{1 *}$ and Thomas J. Brozoski ${ }^{2}$ \\ ${ }^{1}$ Associate Professor, Department of Anatomy and Neurobiology, Northeast Ohio Medical University, Rootstown, OH, United States \\ ${ }^{2}$ Research Professor Emeritus, Division of Otolaryngology, Southern Illinois University School of Medicine, Springfield, IL, United States
}

${ }^{*}$ Corresponding author: Alexander Galazyuk, Associate Professor, Department of Anatomy and Neurobiology, Northeast Ohio Medical University, Rootstown, OH 44272, United States; Email: agalaz@neomed.edu

Received: December 12, 2019; Accepted: December 20, 2019; Published: December 27, 2019

\section{Synopsis}

Animal models have significantly contributed to understanding the pathophysiology of chronic subjective tinnitus. They are useful because they control etiology, which in humans is heterogeneous; employ random group assignment; and often use methods not permissible in human studies. Animal models can be broadly categorized as either operant, or reflexive, based on methodology. Operant methods use variants of established psychophysical procedures to reveal what an animal hears. Reflexive methods do the same using elicited behavior, e.g., the acoustic startle reflex. All methods contrast the absence of sound and presence of sound, since tinnitus cannot by definition be perceived as silence.

Keywords: Animal models, acoustic startle reflex, operant behavioral methods, tinnitus, psychophysics

\section{Key Points}

1. At present there is no standard animal model of tinnitus. Two contemporary types of models are reflexive and operant; each has positive and negative features.

2. Reflexive models trace their origin to an experiment of Turner et al. [1]; operant models trace theirs to an experiment of Jastreboff et al. [2].

3. Caution is advised to distinguish between animal tinnitus studies that independently confirm the presence of tinnitus, and those that do not.

\section{Introduction}

Tinnitus in the present review refers to chronic subjective tinnitus, which has no identifiable acoustic correlate. Despite the common name, "ringing in the ears," its source(s) appear to be primarily in the central nervous system rather than the auditory periphery. Acute tinnitus commonly follows a single exposure to high-level sound or a high dose of aspirin, and typically resolves within minutes to hours. As such it is not of medical concern. In contrast, chronic tinnitus, estimated to affect $35-50$ million adults in the US [3], most commonly follows auditory trauma or chronic hearing loss and often persists for a lifetime[4]. It has been estimated that about five percent of those experiencing chronic tinnitus seek medical treatment. Although common, and recognized since the time of Galen [5], the pathophysiology of tinnitus is incompletely understood. This contributes to the absence of generally effective treatments, although a standard of care has been established $[6,7]$. Tinnitus is typically perceived as a simple sound, a ringing or buzzing sensation, but its pathophysiology is far from simple.

\section{Animal Tinnitus Models}

Tinnitus appears to be a primitive hearing disorder. This is not to say that its pathology is simple, but rather that it derives from basic neurophysiological mechanisms likely to be present in animals as well as humans [8]. Animal models have been available since 1988 [2], and have contributed significantly to understanding the neuroscience of tinnitus $[9,10]$. Although animal models only approximate the human condition, their advantages over clinical studies are several. Most notably: (a) they directly control etiology, (b) they permit application of many experimental tools, from behavioral to molecular, and (c) random assignment to experimental groups enables the use of more powerful inferential statistics as well as attribution of cause. The key problem in developing an animal tinnitus model is objective and reliable assessment, rather than induction. In humans tinnitus can be induced by many conditions. These conditions have in common the reduction of peripheral signal to the brain[11-13].In animals, tinnitus has been induced using systemic treatment with salicylates [2, 14-17], ototoxic exposure [18-20], surgical disruption of the cochlea [21], and acoustic over exposure[19, 22-24]. These methods draw upon factors known to affect tinnitus in humans. The key to solving the assessment problem was provided by Jastreboff and colleagues [25]. Although tinnitus might sound like anything to an animal (or human), it can never sound like silence. All animal models of tinnitus use behavioral methods that differentiate how animals respond to sound versus silence. Typically animal studies also include one or more normalhearing control groups. Although considerable effort has been invested in finding valid and reliable direct measures of tinnitus that do not involve behavior, at present behavioral methods are used exclusively for at least two reasons: There is no procedure for either reliably producing or determining tinnitus alone, without potential confounds. A presumptive tinnitus state might be derived from 
associated phenomena such as hearing loss, hyperacusis, or drug side effects. Behavioral methods enable such confounds to be more clearly sorted out. It should be noted that many presumptive tinnitus animal experiments have examined the effects of conditions likely to cause tinnitus, such as high-level sound exposure or ototoxic damage, without directly confirming the presence of tinnitus. These experiments can be informative about the consequences of auditory insults, but should be interpreted cautiously with respect to tinnitus. Not all humans exposed to acoustic trauma, or other insults develop tinnitus [26]. Similarly it has been shown that not all animals exposed to tinnitusinducing procedures display evidence of tinnitus[27-29]. Therefore, experiments that only examine the consequence of manipulations that typically produce tinnitus, without objective confirmation, are likely to include animals without tinnitus and therefore could be reporting the effects of something other than tinnitus. Unfortunately there is no generally accepted, or standard, animal model of tinnitus against which others can be validated. Existent models have their respective strengths and weaknesses. For overview purposes, animal models can be divided into two broad categories: Models that interrogate animals about their auditory experience, and models that examine alteration of an auditory reflex. Interrogative models, hereafter called operant models, loosely following the terminology of Skinner [30], examine the effect of tinnitus on voluntary, or emitted behavior that is modified by training in an acoustic environment. These models have the general advantage of relying on auditory perception. As such, animals evaluate what they are hearing and differentially respond on the basis of their evaluation. Because operant methods require animals to report what they are hearing, they have conceptual features in common with the interrogation of humans with tinnitus, i.e., analogous to asking "what do you hear?" Operant models tap into functions in many brain areas, including areas outside those commonly defined as auditory. Although this aspect of operant models might be considered a shortcoming, it is also a strength, in that contemporary research has shown tinnitus to be mediated by widely distributed alterations in brain function[11,3134]. A shortcoming of operant models is that they require training and motivating subjects, interventions that can be both time consuming and requiring careful experimental control. In contrast, reflexive animal models rely on unconditioned reflexes, such as the acoustic startle response, and do not require either training or motivation management. Reflexive methods, such as sound gap inhibition of acoustic startle (GPIAS), are also rapid, and therefore well suited to determining the time course of tinnitus development. These features probably account for the current widespread use of GPIAS in animal tinnitus experiments. Although widely used, GPIAS models are not without their own issues and complexities. A further consideration is that the acoustic startle reflex, on which GPIAS is based, depends primarily on brainstem circuits [35]. Therefore the neurophysiological substrate driving the reflexive behavior assessed by GPIAS, might not have the same substrate indicated by operant models.

\section{GPIAS Models}

Animal research: More than ten years ago a new method for tinnitus screening in laboratory animals was introduced by Turner and colleagues [1]. This paradigm utilizes the acoustic startle reflex which is ubiquitously expressed in mammals and consists of contraction of the major muscles of the body following a loud and unexpected sound [36] (Fig.1, A). This reflex is reduced when preceded by a silent gap embedded in a soft background noise or tone (Fig.1, B). Gap detection is typically assessed by the ratio between the magnitude of the startle stimulus presented alone (no-gap trial) and trials in which a gap preceded startle stimulus (gap trials), also known as gap-prepulse inhibition of the acoustical startle (GPIAS) [1]. Reduced inhibition, following acoustic trauma or sodium salicylate treatments is assumed to reflect tinnitus perception: When tinnitus is qualitatively similar to the background noise, it "fills in" the gap and hence, reduces inhibition (Figure 1).

This method was enthusiastically adopted and is now widely used by many scientists in the field due to its relative simplicity over the other methods of tinnitus assessment. Since it is based on a reflex, the method is much cheaper and faster than other methods requiring training animals for weeks or months [22, 59]. It also allows for tinnitus screening of a large number of animals testing simultaneously in multiple testing boxes. Comparing of animals' gap detection performances before and after tinnitus induction allows to separate tinnitus positive from tinnitus negative animals. The possibility of using this method for scientists with little experience in animal behavior and an opportunity to apply this methodology for tinnitus assessment in humans, made GPIAS to dominate in the field of tinnitus research. The GPIAS methodology has been improved upon over the last decade $[37,38]$. It has been shown that careful considerations of GPIAS parameters such as the startle stimulus and background intensities, acoustical parameters of the gap of silence preceding the startle, and overall duration of a testing session, greatly improve results of GPIAS testing in laboratory animals [39].Recent research also demonstrated large variability in GPIAS measurements between different days of testing especially in mice [40]. Therefore averaging these results across multiple testing sessions greatly increases statistical power of the obtained data and improves the reliability of tinnitus assessments. Recent improvements to startle response magnitude assessments [41, 42] and various methods of startle response separation from animals' ambient movements [41, 43] greatly improve GPIAS data analysis. In small rodents the whole body startle reflex is relatively easy to measure, but in larger, less active mammals, such as the guinea pig, it habituates very rapidly. Therefore the pinna reflex measurement technique has been suggested to be used instead of whole body startle reflex during GPIAS sessions $[44,45]$. Despite years of using GPIAS for tinnitus assessment in various laboratory animals, the field continues to debate the original "filling-in" interpretation of the paradigm. In a study conducted on mice, the placement of the gap of silence either closer or further away from the proceeding startle stimulus could dramatically alter gap detection performance in mice [46]. Therefore the authors raised a doubt as to whether tinnitus is "filling-in" the gap, otherwise the gap placement before the startle should not have a large effect on animal's gap detection performance. Importantly however, the most significant debates concerning GPIAS methodology on animals largely depend on successful demonstration that the method is capable of assessing tinnitus in humans. 


\section{Startle response}

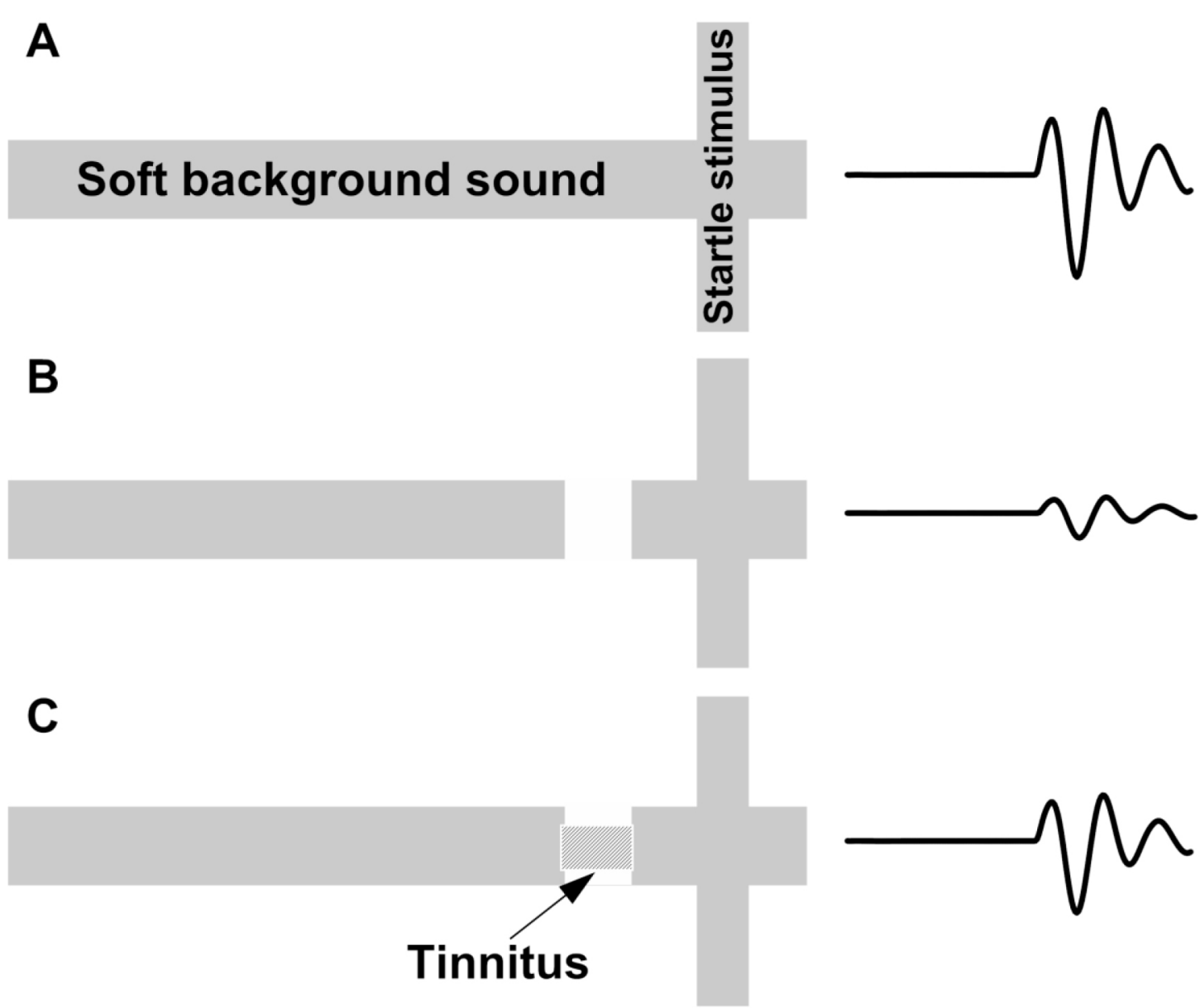

Figure 1. Schematic description of the GPIAS assay for tinnitus. A.A startle wideband noise stimulus 20 ms duration (vertical bar) is inserted into a narrowband noise or pure tone background without gap (no gap; top row) and with a gap (middle and lower rows) 20 to $50 \mathrm{~ms}$ duration and presented $50 \mathrm{~ms}$ before the startle. B.An animal startle responses to the startle stimulus. The response amplitude shown by the height of the startle response waveform (top row). In animals without tinnitus, the gap greatly suppresses the startle response amplitude (middle row). In animals with tinnitus (bottom row), the gap is filled by the tinnitus (shaded rectangular within the gap) and the startle response ismuch less compared to the tinnitus free animals (middle row).

Human research: One of the main advantages of GPIAS over other methods is that it can be used in both laboratory animals and humans [37]. Several research labs have attempted to apply GPIAS method on humans for tinnitus assessment. Eye blink was proposed to be used instead of whole body startle reflex in these studies. These experiments had a significant advantage over the animals' studies because in humans, exact tinnitus parameters such as intensity and spectrum we can identified by tinnitus self-reports. If so, during GPIAS testing it is possible to match the background sound parameters to a person's tinnitus characteristics which would theoretically optimize the success of the GPIAS. Unfortunately, in one of these studies it was found that gap detection performance in tinnitus patients did not depend on whether the individuals have tinnitus or not [47]. Another study showed a difference in dap detection performance between tinnitus patients and controls [48]. However this deficit was not linked to the tinnitus frequency. While these studies raised concerns and emphasized caution, they did not rule out a possibility that GPIAS deficits can indeed be interpreted as an indication of tinnitus.
Indeed, if animals or humans constantly experience a phantom sound, it must still be present during the silent gap during GPIAS testing. Therefore a gap, even partially filled by tinnitus, would still be making gap detection more challenging especially when the background spectrum would closely match the spectrum of tinnitus. Further research on the improvements of GPIAS testing paradigm might help to detect gap detection challenges in tinnitus patients. The most recent work has attempted to directly measure human neurophysiological correlates of gap detection with cortical auditory evoked potentials (CAEP) recorded in the electroencephalogram (EEG) [49]. The N1 potentials in response to gaps of silence were recorded from scalp in normal tinnitus-free individuals. Such an approach does not require overt responses from the participant nor measures responses modulated by gaps. Gap-evoked cortical responses were identified in all conditions for the vast majority of participants. The N1 responses were independent on background noise frequencies or background levels. The authors recommend that this experimental design could be used in both animals and humans to identify tinnitus objectively. 


\section{Early Operant Models}

A variety of operant methods for tinnitus determination in animals have been developed. Two early operant models, those developed by Jastreboff et al. [2] and Bauer et al. [22],illustrate many features common to these models. Operant models examine the effect of tinnitus on emitted behavior that has been modified by auditory training. Both methods are interrogative, in that they require subjects to respond differentially to auditory events. In the Jastreboff model, tinnitus was induced by high systemic doses of sodium salicylate. Rats were conditioned to stop licking a water spout by imposing a mild electric shock, at the end of random periods when the background sound (broad-band noise; BBN, $60 \mathrm{~dB}$, SPL) was turned off, i.e., external silence. The animals were then tested with randomly-inserted silent periods, without shocks, following acute salicylate exposure $(300 \mathrm{mg} / \mathrm{kg})$. The salicylate-treated animals showed more persistent licking during the sound-off periods than controls without salicylate [2]. The interpretation was that salicylate induced tinnitus, as it is well known to do in humans, and masked the sound-off silence; therefore the rats continued to lick as they would have if sound were present. In an informative variant procedure, Jastreboff et al. demonstrated the obverse effect with animals that were lick-suppression trained while on salicylate [2]. In this variant, the rats suppressed licking more during sound-off test periods than non-salicylate controls. The interpretation was that suppression training, with tinnitus present, conditioned the animals to not lick when their tinnitus, a salient internal sound, was heard. A limitation of the Jastreboff salicylate lick-suppression model is that it was only suitable for determining acute tinnitus. Reasons for this limitation are twofold: tinnitus induced by salicylate treatment is temporary, subsiding within a day or so after discontinuing the drug, and more importantly, the tinnitus influence on licking was measured during extinction of conditioned suppression (there were no shocks when tinnitus testing).Extinction is a transient behavioral state.

A derivative operant method, well suited to assessing chronic tinnitus and still in use, was developed by Bauer and colleagues[14 22 23]. In the Bauer model, chronic tinnitus was induced using a single unilateral exposure to moderate-level tones ( $4 \mathrm{kHz}$ at $80 \mathrm{~dB}$ SPL) in chinchillas, or high-level band-limited noise centered at $16 \mathrm{kHz}(116$ - $120 \mathrm{~dB}, \mathrm{SPL}$ ) in rats, for one hour, three or more months prior to tinnitus assessment. Unilateral exposure was used to preserve normal hearing in one ear. It also reflects a condition commonly associated with tinnitus in humans. Asymmetric acoustic trauma or hearing loss in humans is commonly associated with chronic tinnitus, including bilateral tinnitus [50]. All animals were trained to lever press for food pellets in the presence of broadband noise (BBN) (60 dB, SPL) and were tested for tinnitus using randomly introduced 1-min periods of either sound off, or tones at various levels. Lever pressing during sound-off periods was suppressed by delivering a lever-presscontingent foot shock at the end of sound-off periods. In other words, the animals could avoid the foot shock by not lever pressing during sound off. Tinnitus was indicated by decreased lever pressing when tested with tones in the vicinity of $20 \mathrm{kHz}$ (Fig 2A), although tones of various frequency at various levels were tested. Control animals were not exposed to tinnitus induction but were otherwise treated and tested in parallel. The interpretation was that animals with chronic tinnitus could not hear true silence, but instead heard their tinnitus. Because they were trained to suppress lever pressing when their tinnitus was audible (during sound off periods), they suppressed lever pressing to stimulus-driven sensations that resembled their tinnitus[8, 22].Note that in the Bauer model testing and training are integrated into every session. This meant that chronic tinnitus could be measured with undiminished sensitivity over long periods. The model has been used to assess tinnitus in rats for as long as 17 months [22]. It was also found that a proportion of the exposed animals, typically 30 to 40 percent, did not develop tinnitus, although the audiometric profile of all exposed animals was equivalent (Fig. 2B).The Bauer model has also been used to determine acute tinnitus induced by systemic salicylate [14] as well as chronic tinnitus induced by ototoxic exposure [19]. (Figure 2)

\section{Operant Model Variations}

Experimenters have examined a number of variations in an attempt to improve operant models. Several excellent reviews of tinnitus models may be consulted for variant features [51-53]. The extended training required by the Bauer model negatively impacts throughput, and can be shortened by employing an unconditioned indicator such as licking a spout for water. A number of researchers have adopted this modification. Zheng and colleagues developed a model that incorporated many features of the Bauer model, using water deprived rats required to lick a spout for water instead of pressing a lever for food [54]. This considerably decreased training time, although it did not decrease the time required for tinnitus to appear after acoustic induction. A wrinkle that must be addressed when substituting licking for lever pressing is the episodic nature of licking. Spontaneous pauses in licking must be taken into account, so as notto count themas false positive suppressions. Zheng et al., used shortened test sessions to reduce this error. In another operant variation, using licking behavior, May and colleagues trained rats to lick to sound resembling their tinnitus, rather than suppressing to tinnitus-like sound [55]. Chronic tinnitus was induced using high-level sound exposure while acute tinnitus was induced using high-dose salicylate treatment. Episodic features of licking were controlled by using test periods of only a few minutes, and by using a tinnitus score normalized to each animal's non-test lick rate. They found acoustic-induced chronic tinnitus with characteristics similar to $16 \mathrm{kHz}$ tones, while acute salicylate induced tinnitus was similar to narrow-band noise between 8 and $22 \mathrm{kHz}$. Licking in combination with conditioned place preference has been used to indicate chronic acoustic-induced tinnitus in hamsters [56]. Two spouts were available from which to drink, each in a distinct location; animals were trained to use the non-preferred spout in the presence of an ipsi lateral external sound. Testing occurred in silence. Licking at the sound-conditioned (non-preferred) spout indicated tinnitus [29]. Using a variant of this method, Heffner trained rats to lick from visual-and-auditory cued left or right water spouts. After unilateral sound exposure, Heffner was able to use left vs right spout choice to indicate tinnitus lateral localization [57]. This informative experiment demonstrates how operant methods have been adapted to answer specific questions, such as tinnitus laterality. 

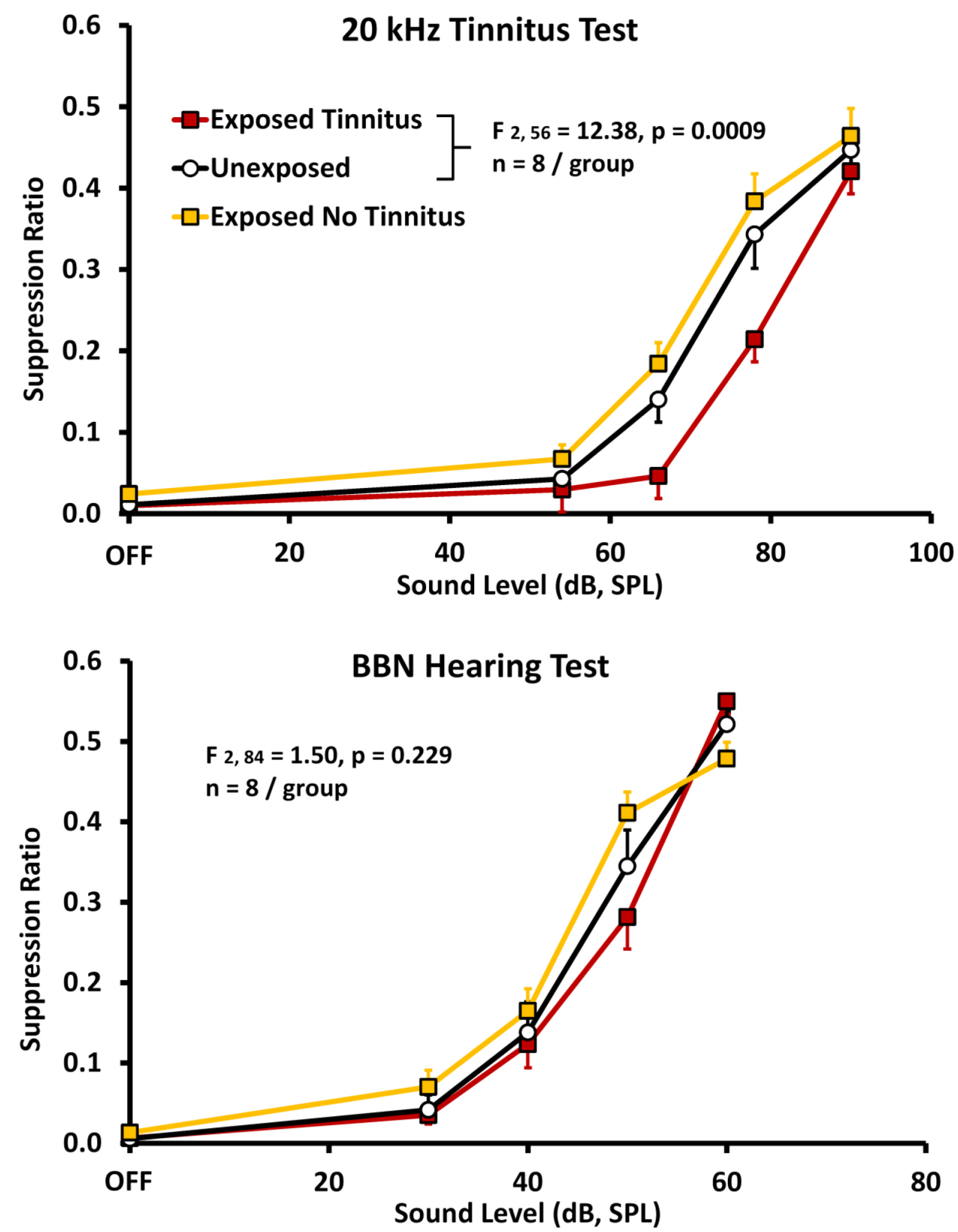

Figure 2. Psychophysical discrimination functions obtained from three groups of rats; relative lever pressing, recorded as a suppression ratio (y-axis) is plotted against test-stimulus sound level (x-axis). A suppression ratio of 0 reflects no lever presses, while a suppression ratio of 0.5 reflects lever pressing at baseline rate preceding the test stimulus. Both experimental groups $(\mathrm{n}=8 \mathrm{each}$; filled square data points) were unilaterally exposed to band-limited noise ( $120 \mathrm{~dB}$, SPL, octave band centered at 16 $\mathrm{kHz})$ six months prior to testing. The unexposed controls $(\mathrm{n}=8$; unfilled circular data points) were trained and tested in parallel. Panel A shows the average of 5 sessions using $20 \mathrm{kHz}$ test tones. A subset of exposed subjects suppressed significantly more to the $20 \mathrm{kHz}$ stimuli. The statistical difference between the Exposed-with-tinnitus and Unexposed groups is shown in the inset. Suppression behavior (average of 5 sessions) of the same animals tested with broad band noise (BBN), diagnostic for free-field hearing but not tinnitus, is shown in panel B. Data points are group means averaged over 5 test sessions; error bars indicate the standard error of the mean. Significance levels were determined using a mixed analysis of variance ( $\mathrm{n}=8$ per group). SPL, sound pressure level. 


\section{Model Features: Pros and Cons}

Using licking as an indicator requires water restriction, typically for 24 hrs. A nontrivial consideration is the physiological stress imposed by water deprivation. It has been shown that restricting water intake in rodents for $24 \mathrm{hrs}$ leads to vasopressin and vascular-induced central neural changes that are reflected in physiological stress indicators and behavioral dysfunction [58]. An interesting lick suppression method not requiring water restriction, and its attendant physiological stress, was developed by Lobarinas and colleagues [59]. The motivation to lick for water was induced in rats by delivering "free" food pellets at regular intervals. Although the animals had to be food deprived, they did not have to be water deprived or extensively trained to lick. Since rats are prandial drinkers, distributed food delivery will induce licking, hence schedule-induced polydipsia (SIPAC). Once SIPAC stabilizes, licking can be suppressed to an acoustic signal, using an electric shock. Sound-off licking can then be compared between animals with tinnitus and those without, with the expectation that tinnitus animals will do less sound-off licking than non-tinnitus controls because their tinnitus provides an auditory signal for suppression. Variability of performance over time and between subjects, however, has been an issue for this model [51]. Unlike reflex-based animal models, operant models are obliged to motivate subjects to respond appropriately to sensory events. As some pet owners and all animal trainers know, animals will not comply with human requests unless they are motivated. Typically motivation is experimentally established by restricting access to food or water, or by imposing an aversive stimulus. These three strategies may be employed singularly or in combination to comprise a given method. Operant models described so far have in common the combined use of positive reinforcement, such as food or water, and punishment procedures, such as foot shock. It is well known that aversive stimulus control lends itself to more rapid conditioning than positive control [60]. With that in mind, some animal models have exclusively used aversive stimulus control to improve efficiency. Guitton et al., trained rats to jump from an electrified floor to an insulated pole when an auditory signal was present [61]. Since the task was moderately strenuous, the animals had a low spontaneous rate of jumping without foot shock. After salicylate treatment the animals were tested without sound and spontaneous pole jumps were recorded; an elevated number of jumps indicated tinnitus. Using this model, both group and individual comparisons could be made, with animals serving as their own control. A limitation was that the method does not lend itself cleanly to testing chronic tinnitus, and as a discrete-trial procedure the animals typically had to be handled between trials in order for a new trial to be initiated. Handling introduces a potential source of error that may not be entirely controlled by treatment blinding, since an increased number of spontaneous jumps would un-blind the experimenter. Relying exclusively on aversive control also interjects a stress factor. However stress could be considered a positive feature, since humans frequently comment that stress exacerbates their tinnitus.

\section{Summary}

\section{Features of an Ideal Animal Tinnitus Model}

Criteria of validity, sensitivity, and reliability have to be balanced against efficiency, cost, and throughput, in any animal model. An ideal model would be sensitive enough to detect low levels of tinnitus, yet clearly separate tinnitus from confounds such as hearing loss and hyperacusis. The sensitivity of an ideal model would not diminish with repeated testing, allowing measurement of chronic tinnitus and the use of extended test series necessary to test therapeutics. Sensitive and reliable models should also require a low number of animals. Determining validity is never as clear cut as determining reliability; however animal models should be validated against one another and against quantitative human data whenever possible. Tinnitus features such as pitch, loudness, and duration should be reflected in all models. Finally, a more direct, and ideally noninvasive, measure of tinnitus, not requiring extended psychophysical testing would be very advantageous.

\section{Acknowledgement}

Preparation of this manuscript was supported by research grantR01 DC016918 from the National Institute on Deafness and Other Communication Disorders of the U.S. Public Health Service (A.V.G.)

\section{References}

1. Turner JG, Brozoski TJ, Bauer CA, Parrish JL, Myers K et al. (2006) Gap detection deficits in rats with tinnitus: a potential novel screening tool. Behav Neurosci 120 : 188-195. [Crossref]

2. Jastreboff PJ, Brennan JF, Coleman JK, Sasaki CT (1988) Phantom auditory sensation in rats: an animal model for tinnitus. Behav Neurosci 102: 811-822. [Crossref]

3. Shargorodsky J, Curhan GC, Farwell WR (2010) Prevalence and characteristics of tinnitus among US adults. The American journal of medicine 123: 711-718. [Crossref]

4. Bauer CA (2018) Tinnitus. N Engl J Med 378: 1224-1231. [Crossref]

5. Dan B (2005) Titus's tinnitus. J Hist Neurosci 14: 210-213. [Crossref]

6. Tunkel DE, Bauer CA, Sun GH, Rosenfeld RM, Chandrasekhar SS et al. (2014) Clinical practice guideline: tinnitus executive summary. Otolaryngol Head Neck Surg 151: 533-541. [Crossref]

7. Tunkel DE, Jones SL, Rosenfeld RM (2016) Guidelines for Tinnitus. JAMA 316: 1214-1215. [Crossref]

8. Brozoski TJ, Bauer CA (2008) Learning about tinnitus from an animal model. Seminars in Hearing 29: 242-258.

9. Kaltenbach JA (2011) Tinnitus: Models and mechanisms. Hear Res 276: 52-60. [Crossref]

10. Brozoski TJ, Bauer CA (2016) Animal models of tinnitus. Hear Res 338: 88-97. [Crossref]

11. Norena AJ, Farley BJ (2013) Tinnitus-related neural activity: theories of generation, propagation, and centralization. Hear Res 295: 161-171. [Crossref]

12. Schaette R (2014) Tinnitus in men, mice (as well as other rodents), and machines. Hear Res 311: 63-71. [Crossref]

13. Yang S, Bao S (2013) Homeostatic mechanisms and treatment of tinnitus. Restorative neurology and neuroscience 31: 99-108. [Crossref]

14. Bauer CA, Brozoski TJ, Rojas R, Boley J, Wyder M (1999) Behavioral model of chronic tinnitus in rats. Otolaryngol Head Neck Surg 121: 457-62. [Crossref]

15. Mahlke C, Wallhausser-Franke E (2004) Evidence for tinnitus-related plasticity in the auditory and limbic system, demonstrated by arg3.1 and c-fos immunocytochemistry. Hear Res 195: 17-34. [Crossref]

16. Ruttiger L, Ciuffani J, Zenner HP, Knipper M (2003) A behavioral paradigm to judge acute sodium salicylate-induced sound experience in rats: a new approach for an animal model on tinnitus. Hear Res 180: 39-50. [Crossref]

17. Yang G, Lobarinas E, Zhang L, Turner J, Stolzberg D et al. (2007) Salicylate induced tinnitus: behavioral measures and neural activity in auditory cortex of awake rats. Hear Res 226: 244-253. [Crossref]

18. Alkhatib A, Biebel UW, Smolders JW (2006) Reduction of inhibition in the inferior colliculus after inner hair cell loss. Neuroreport 17: 1493-1497. [Crossref]

19. Bauer CA, Turner JG, Caspary DM, Myers KS, Brozoski TJ (2008) Tinnitus and inferior colliculus activity in chinchillas related to three distinct patterns of cochlear trauma. J Neurosci Res 86: 2564-2578. [Crossref] 
20. Kaltenbach JA, Rachel JD, Mathog TA, Zhang J, Falzarano PR et al. (2002) Cisplatin-induced hyperactivity in the dorsal cochlear nucleus and its relation to outer hair cell loss: relevance to tinnitus. J Neurophysiol 88: 699-714. [Crossref]

21. Zacharek MA, Kaltenbach JA, Mathog TA, Zhang J (2002) Effects of cochlear ablation on noise induced hyperactivity in the hamster dorsal cochlear nucleus: implications for the origin of noise induced tinnitus. Hear Res 172: 137-143. [Crossref]

22. Bauer CA, Brozoski TJ (2001) Assessing tinnitus and prospective tinnitus therapeutics using a psychophysical animal model. $J$ of the Assoc for Res in Otolaryngol 2: 54-64. [Crossref]

23. Brozoski TJ, Bauer CA, Caspary DM (2002) Elevated fusiform cell activity in the dorsal cochlear nucleus of chinchillas with psychophysical evidence of tinnitus. $J$ Neurosci 22: 2383-2390. [Crossref]

24. Dehmel S, Pradhan S, Koehler S, Bledsoe S, Shore S (2012) Noise overexposure alters long-term somatosensory-auditory processing in the dorsal cochlear nucleus-possible basis for tinnitus-related hyperactivity?. J Neurosci 32: 1660-1671. [Crossref]

25. Jastreboff PJ, Brennan JF, Sasaki CT (1988) An animal model for tinnitus. Laryngoscope 98: 280-286. [Crossref]

26. Tyler RS, Erlandsson S (2000) Management of the tinnitus patient. In:Luxon LM (ed.). Textbook of audiological medicine. Oxford: Isis, Pg 571-578.

27. Ahlf S, Tziridis K, Korn S, Strohmeyer I, Schulze H (2012) Predisposition for and prevention of subjective tinnitus development. PLoS One 7. [Crossref]

28. Bauer CA, Wisner K, Sybert LT, Brozoski TJ (2013) The cerebellum as a novel tinnitus generator. Hear Res 295:130-139. [Crossref]

29. Heffner HE, Harrington IA (2002) Tinnitus in hamsters following exposure to intense sound. Hear Res 170: 83-95. [Crossref]

30. Skinner BF (1938) The behavior of organisms; an experimental analysis. New York, London,: D. Appleton-Century Company .

31. Eggermont JJ, Roberts LE (2004) The neuroscience of tinnitus. Trends Neurosci 27: 676-82. [Crossref]

32. Roberts LE, Eggermont JJ, Caspary DM, Shore SE, Melcher JR, et al. (2010) Ringing ears: the neuroscience of tinnitus. J Neurosci 30: 14972-14979. [Crossref]

33. Brozoski TJ, Ciobanu L, Bauer CA(2007) Central neural activity in rats with tinnitus evaluated with manganese-enhanced magnetic resonance imaging (MEMRI). Hear Res 228: 168-179. [Crossref]

34. Brozoski TJ, Wisner KW, Odintsov B, Bauer CA (2013) Local NMDA receptor blockade attenuates chronic tinnitus and associated brain activity in an animal model. PLoS One 8. [Crossref]

35. Gomez-Nieto R, Horta-Junior Jde A, Castellano O, Millian-Morell L, Rubio ME et al. (2014) Origin and function of short-latency inputs to the neural substrates underlying the acoustic startle reflex. Frontiers in neuroscience 8. [Crossref]

36. Koch M (1999) The neurobiology of startle. Progress in Neurobiology 59: 107-128. [Crossref]

37. Galazyuk A, Hébert S (2015) Gap-Prepulse Inhibition of the Acoustic Startle Reflex (GPIAS) for Tinnitus Assessment: Current Status and Future Directions. Front Neurol 6. [Crossref]

38. Shore SE, Roberts LE, Langguth B (2016) Maladaptive plasticity in tinnitus-triggers, mechanisms and treatment. Nat Rev Neurol 12: 150-60. [Crossref]

39. Longenecker R, Galazyuk AV (2012) Methodological optimization of tinnitus assessment using prepulse inhibition of the acoustic startle reflex. Brain Res 1485 : 54-62. [Crossref]

40. Longenecker RJ, Galazyuk AV (2016) Variable effects of acoustic trauma on behavioral and neural correlates of tinnitus in individual animals. Front Behav Neurosci 10. [Crossref]

41. Grimsley CA, Longenecker RJ, Rosen MJ, Young JW, Grimsley JM, et al. (2015) An improved approach to separating startle data from noise. J Neurosci Methods 253: 206-217. [Crossref]

42. Gerum R, Rahlfs H, Streb M, Krauss P, Grimm J et al. (2019) Open(G)PIAS: r tinnitus screening and threshold estimation in rodents. Front Behav Neurosci 13. [Crossref]

43. Choe Y, Park I (2019) Proposal of conditional random interstimulus interval method for unconstrained enclosure based GPIAS measurement systems. Biomed Eng Let 9: 367-374.

44. Berger JI, Coomber B, Shackleton TM, Palmer AR, Wallace MN (2013) A novel behavioural approach to detecting tinnitus in the guinea pig. $J$ Neurosci Methods 213: 188-95. [Crossref]

45. Wu C, Martel DT, Shore SE (2016) Increased Synchrony and Bursting of Dorsal Cochlear Nucleus Fusiform Cells Correlate with Tinnitus. J Neurosci 36: 20682073. [Crossref]

46. Hickox AE Liberman MC (2014) Is noise-induced cochlear neuropathy key to the generation of hyperacusis or tinnitus? J Neurophysiol 111: 552-564. [Crossref]
47. Fournier P, Hébert S (2013) Gap detection deficits in humans with tinnitus as assessed with the acoustic startle paradigm: does tinnitus fill in the gap? Hearing research 295: 16-23. [Crossref]

48. Gilani VM, Ruzbahani M, Mahdi P, Amali A, Khoshk MHN, et al. (2013) Temporal processing evaluation in tinnitus patients: results on analysis of gap in noise and duration pattern test. Iranian journal of otorhinolaryngology 25: 221-226. [Crossref]

49. Paul BT, Schoenwiesner M, Hébert S (2018) Towards an objective test of chronic tinnitus: Properties of auditory cortical potentials evoked by silent gaps in tinnitus-like sounds. Hear Res 366: 90-98. [Crossref]

50. Davis A, El Refaie (2000) A Epidemiology of Tinnitus. In: Tyler RS (ed.). Tinnitus Handbook. Clifton Park, NY: Delmar Cengage Learning, Pg 1-24.

51. Hayes SH, Radziwon KE, Stolzberg DJ, Salvi RJ (2014) Behavioral models of tinnitus and hyperacusis in animals. Front Neurol 5. [Crossref]

52. von der Behrens W (2014) Animal models of subjective tinnitus. Neural Plast 2014. [Crossref]

53. Stolzberg D, Salvi RJ, Allman BL (2012) Salicylate toxicity model of tinnitus. Front Syst Neurosci 6. [Crossref]

54. Zheng Y, Vagal S, McNamara E, Darlington CL, Smith PF (2012) A dose-response analysis of the effects of L-baclofen on chronic tinnitus caused by acoustic trauma in rats. Neuropharmacology 62: 940-946. [Crossref]

55. Jones A, May BJ (2017) Improving the Reliability of Tinnitus Screening in Laboratory Animals. J Assoc Res Otolaryngol 18: 183-195. [Crossref]

56. Heffner HE, Koay G (2005) Tinnitus and hearing loss in hamsters (Mesocricetus auratus) exposed to loud sound. Behav Neurosci 119: 734-742. [Crossref]

57. Heffner HE (2011) A two-choice sound localization procedure for detecting lateralized tinnitus in animals. Behav Res Methods 43: 577-589. [Crossref]

58. Faraco G, Wijasa TS, Park L, Moore J, Anrather J (2014) Water deprivation induces neurovascular and cognitive dysfunction through vasopressin-induced oxidative stress. Journal of cerebral blood flow and metabolism : official journal of the International Society of Cerebral Blood Flow and Metabolism 34: 852-860. [Crossref]

59. Lobarinas E, Sun W, Cushing R, Salvi R (2004) A novel behavioral paradigm for assessing tinnitus using schedule-induced polydipsia avoidance conditioning (SIPAC). Hear Res 190: 109-114. [Crossref]

60. LeDoux JE (2000) Emotion circuits in the brain. Annu Rev Neurosci 23: 155-'184. [Crossref]

61. Guitton MJ, Caston J, Ruel J, Johnson RM, Pujol R, et al. (2003) Salicylate induces tinnitus through activation of cochlear NMDA receptors. J Neurosci 23: 3944 3952. [Crossref]

\section{Citation:}

Alexander Galazyuk, Thomas J. Brozoski (2019) Animal Models of Tinnitus: A Review. Otorhinolaryngol Hypersensitivity Treat Volume 1(1): 1-7. 